\title{
Relationship between population characteristics, e-cigarette and tobacco-related perceptions, and likelihood of ever using e-cigarettes
}

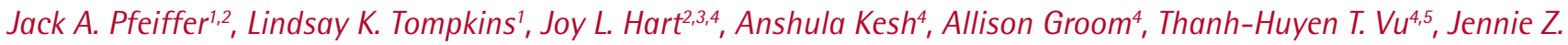 \\ Ma ${ }^{4,6}$, Robyn Landry', Thomas J. Payne ${ }^{4,7}$, Aida L. Giachello ${ }^{4,8}$, Rose Marie Robertson ${ }^{4}$, Kandi L. Walker ${ }^{2,3,4}$
}

\begin{abstract}
INTRODUCTION Electronic nicotine delivery systems (ENDS) are a relatively new type of nicotine-containing product that has risen greatly in use within the past decade, displacing conventional tobacco products as the dominant source of nicotine exposure by many groups. Among those impacted are large sections of US youth. Though health outcomes associated with ENDS use are still being assessed, several potential harms have been noted in the extant literature. The purpose of this study is to examine which US youth subpopulations are at greatest risk for ENDS ever use and how perceptions pertaining to nicotine-containing products relate to this risk.

METHODS A nationwide online survey was administered to US youth ENDS users and non-users aged 13-18 years. A total weighted sample of 2501 participants was obtained. Statistical analyses included binomial logistic regression and a likelihood ratio test.

RESULTS Of these youth, 1346 (53.8\%) reported having ever used an ENDS product. Those most likely to have used ENDS were White males in their late teens. Those who reported ever using a conventional tobacco product were much more likely to have reported ever using ENDS (AOR=19.96; 95\% CI: 15.30-26.05). A number of perceptions related to nicotine-containing products, including product safety and health effects, were significantly associated with an increased likelihood of ENDS use.

CONCLUSIONS Certain sections of the US youth population have elevated odds of being ENDS ever users. As increasing evidence supports the need to combat ENDS use by youth, effectively targeted education and prevention campaigns will be necessary.
\end{abstract}

\author{
AFFILIATION \\ 1 Department of Epidemiology, University \\ of Louisville, Louisville, United States \\ 2 Christina Lee Brown Envirome Institute, \\ University of Louisville, Louisville, United \\ States \\ 3 Department of Communication, \\ University of Louisville, Louisville, United \\ States \\ 4 American Heart Association, Tobacco \\ Regulation and Addiction Center, Dallas, \\ United States \\ 5 Department of Preventative Medicine \\ (Epidemiology), Feinberg School of \\ Medicine, Northwestern University, \\ Chicago, United States \\ 6 Division of Biostatistics, University of \\ Virginia, Charlottesville, United States \\ 7 Department of Otolaryngology and \\ Communicative Sciences, University of \\ Mississippi Medical Center, Jackson, United \\ States \\ 8 Department of Preventative Medicine \\ (Public Health Practice), Feinberg School \\ of Medicine, Northwestern University, \\ Chicago, United States
}

\section{CORRESPONDENCE TO}

Joy L. Hart. Christina Lee Brown Envirome Institute, University of Louisville, Louisville, $\mathrm{KY}$, United States.

E-mail: joy.hart@louisville.edu

\section{KEYWORDS}

tobacco, electronic cigarette, vaping, tobacco perceptions, risk of tobacco use, e-nicotine

Received: 10 November 2019

Revised: 25 January 2020

Accepted: 26 January 2020

\section{INTRODUCTION}

Despite numerous years of anti-tobacco campaigns, including US-based efforts such as the American
Legacy Foundation 'Truth Initiative', the Centers for Disease Control and Prevention (CDC) 'Tips From Former Smokers', and the Food and Drug 
Administration (FDA) 'The Real Cost', use of conventional tobacco continues to be the largest preventable source of morbidity and mortality worldwide ${ }^{1-4}$. An estimated 0.48 million people die prematurely each year in the US due to smoking conventional cigarettes and exposure to secondhand smoke. Conventional cigarette use, as well as use of other forms of conventional tobacco, are on the decline. The CDC has estimated that the number of current adult conventional cigarette users has dropped from about $42.4 \%$ in 1965 to $15.5 \%$ in $2016^{15,16}$. Further reports reveal that the number of current youth conventional cigarette users has also dropped considerably, from $4.3 \%$ in 2011 to $1.8 \%$ in 2018 among middle school students, and from $15.8 \%$ in 2011 to $8.1 \%$ in 2018 among high school students ${ }^{7,8}$. As conventional tobacco use declines, other methods of nicotine consumption have risen, most notably electronic nicotine delivery systems (ENDS) $)^{9,10}$.

Originally developed in the early $2000 \mathrm{~s}^{11}$, ENDS first arrived in the US marketplace in 2007 in the form of electronic cigarettes ${ }^{12,13}$. Since then, numerous other electronic nicotine products have emerged. ENDS comprise a group of products that include e-cigarettes, vape pens, hookah pens, personalized vaporizers and mods, e-cigars, e-pipes, and e-hookahs. As ENDS evolved, longlasting batteries and designs allowing for use of an assortment of flavored e-liquids have become common. These e-liquids are heated, producing an aerosol that is inhaled. Although some e-liquids do not contain nicotine, many do, and the nicotine content of these liquids can vary greatly and is not currently subject to strict government oversight.

The scientific community is divided on the potential benefits and harms of using ENDS, with some research findings mixed and too little time having passed to amass a body of research on long-term outcomes. Advocates for ENDS suggest that they can be useful tools for the cessation of conventional cigarette use, especially as the use of ENDS closely mimics the hand-mouth motions associated with smoking conventional cigarettes ${ }^{13}$. Further, in some studies, ENDS use has been found to help reduce cigarette cravings in adult smokers and reduce the likelihood of relapse in those who are in the process of quitting ${ }^{14,15}$. Additionally, some researchers note that exposure to secondhand
ENDS aerosol differs from exposure to secondhand smoke $^{13}$.

Despite the possibility of some beneficial uses, the exact nature of ENDS toxicity, potential benefits from use and associated health concerns have not been thoroughly elucidated. A growing body of literature suggests that ENDS are not as innocuous as once thought ${ }^{16}$. Increasing evidence indicates that ENDS use is not an effective means of quitting combustible cigarettes and is associated with increased levels of conventional tobacco use initiation and dual use among youth ${ }^{10,17}$. Even when ENDS have been shown to be more effective than traditional nicotine-replacement therapy (NRT) for the cessation of smoking, those who used ENDS as their means of quitting were much more likely to maintain usage at one year of smoking abstinence than those who used NRT ${ }^{18}$. Further, ENDS use may be predictive of future cigarette use in high school youth, and continued use of ENDS across time may result in increased frequency of consumption ${ }^{19}$. Despite the dangers, ENDS are aggressively marketed to adolescents through targeted marketing schemes, often touting varied flavor options and potentially resulting in adolescent use of nicotine products that might not have occurred otherwise ${ }^{9,12,17}$. More recently, cases of e-cigarette or vaping product use-associated lung injury (EVALI), resulting in several deaths and hospitalizations, have led the CDC to recommend against using ENDS and to emphasize that youth should never use these products $^{20}$.

It is not yet clear whether the same populations considered vulnerable to conventional tobacco use are equally affected by ENDS use ${ }^{21}$. In studies to date, ENDS use has been consistently associated with being male and non-Hispanic White as well as with increasing age among youth ${ }^{22-25}$. It is clear that youth are susceptible to initiating ENDS use, given the substantial rise in prevalence in recent years. The National Youth Tobacco Survey (NYTS) findings noted an increase in ENDS ever use among middle schoolers from $1.4 \%$ in 2011 to $4.9 \%$ in 2018, and an increase in ENDS ever use among high schoolers from $4.7 \%$ in 2011 to $20.8 \%$ in $2018^{7,9}$. In 2018, ENDS were the most commonly used nicotine-containing product among middle and high schoolers? 
The purpose of this study is to elucidate the relationship between population characteristics, perceptions of nicotine-containing products, and the likelihood of initiating ENDS use among US youth aged 13-18 years. In doing so, susceptible populations may be revealed, and ENDS education efforts can be correspondingly targeted in a more efficient and effective manner.

\section{METHODS}

Between August and October 2017, an online survey of US youth ENDS users and non-users, aged 1318 years, was conducted. Ypulse, a research and marketing firm specializing in preteen, teen, college student, and young adult panels, fielded the survey. To obtain data, Ypulse manages an incentive-based online research panel called SurveyU, with approximately 65000 members, as well as recruits through buzz campaigns, ads in newspapers, and social networking.

Study methods and materials were approved for use by the Chesapeake/Advarra Institutional Review Board (IRB), and privacy guidelines outlined in the Children's Online Privacy Protection Act (COPPA) were followed. Participation in the survey was voluntary. Parental/guardian consent was required for participants under the age of 18 years, with those participants completing assent forms. Participants who were 18 years old completed consent forms. De-identification of data was not necessary, as no identifying information was gathered.

The study consisted of 3174 participants. Two groups were recruited: a) ENDS users, defined as youth who had ever tried e-cigarettes or other ENDS, with a subset of dual users (had tried ENDS and another tobacco product); and b) a control group, defined as youth who had never tried ENDS. Quotas were established for participant recruitment based on gender, age, race, and ethnicity. Non-Hispanic Black and Hispanic individuals were oversampled to ensure sufficient sample sizes for comparisons by ethnicity and race. Researchers tracked age, gender, and race/ethnicity across respondents to enable accurate weighting of the results. Post hoc blocking was used to evaluate other issues of interest, and the data were weighted to be representative of the overall US population. Weighting was based on 2017 US Census data. Proportions for each group to be weighted were calculated using census data along with corresponding proportions from the study sample, which allowed the calculation of the final weight.

Of the 3174 total survey participants, 2654 participants were included in the analytic sample after accounting for missing observations and prior to weighting. Missing observations were removed from the variables ethnicity, parent education level, participant education level, race, sexual orientation, and use of lunch assistance programs. Specifically, missing data were: 125 observations for ethnicity due to participants indicating 'don't know' when asked if they identified as Hispanic; 219 observations for parent education due to participants answering 'not sure' when asked about parent/guardian education level; 7 observations for participant education due to inadequate responses to the 'other' option; 88 observations for race due to participants answering 'don't know' when asked about their racial identity; 32 observations for sexual orientation due to participants indicating "prefer not to answer'; 176 observations for lunch program due to participants answering 'not sure' when asked if they were currently utilizing a financial assistance program for school lunch. After weighting, the sample population was composed of 2501 youth. The final sample size after weighting was lower due to the weight being less than one. Non-users served as the control group. All results are based upon the weighted sample. Unweighted data are provided for reference.

\section{Measures}

\section{Tobacco use status}

ENDS use status was defined based upon responses to the question: "Which of the following types of tobacco have you ever tried (even one time or two times)?'. Eleven response categories were provided, each with the opportunity to answer either 'yes' or 'no'. The categories were: electronic nicotine products, conventional cigarettes, conventional cigars, little or filtered cigars, cigarillos, smokeless tobacco, dissolvable tobacco, hookah, bidis and kreteks, other, and never used. Product examples and images were provided for all but the last two categories. Those who reported ever using an electronic nicotine product were categorized as ENDS ever users, and those who did not report ever using an electronic 
nicotine product were categorized as ENDS never users. Participants who had ever used any non-ENDS nicotine product were grouped together into a single conventional tobacco ever use variable.

\section{Sociodemographic variables}

Several variables were taken into consideration to determine which were associated with ENDS use among youth. Sociodemographic variables included age, household size, living status, parental education level, participant education level, race, ethnicity, gender, sexual orientation, residence location, and use of lunch assistance programs.

Age was defined in years. Household size was defined by the number of people living in the participant's household. Living status was defined as living with both parents, one parent, an alternative guardian or caretaker, or none of the previous. Parental education level was split into six subgroups: less than a high school education, high school graduate or GED, some college, Associate's or technicial degree, Bachelor's degree, and graduate or professional degree. Participant education level was subdivided into three categories: being in middle school or less, in high school, and a high school graduate or greater. Race and ethnicity were combined to form a single variable with four categories: Non-Hispanic White, Non-Hispanic Black, Hispanic, and Non-Hispanic Other. The Non-Hispanic Other category included those who indicated their race as Asian/Pacific Islander, American Indian, other, or multiple races. Gender was defined as female or male. Sexual orientation was defined as straight or LGBTQ. Residence location was classified as urban, suburban, or rural. Use of lunch assistance programs was defined as either yes or no, depending on whether the participant currently utilized lunch assistance resources at school.

\section{Tobacco use health and safety}

Participants were prompted to answer four ordinal scale items that provided information about perceived safety and risk involved with tobacco and ENDS use. The items were: 'How knowledgeable are you about health risks linked to tobacco use?'; 'How much do you know about health risks linked to using electronic nicotine products?'; 'How safe or dangerous do you think electronic nicotine products are?'; and 'How safe or dangerous do you think electronic nicotine products are compared with other tobacco products?'. The first three items were answered using a scale of $0-10$, with the first two items ranging from 'not at all' to 'very much' and the third ranging from 'very dangerous' to 'very safe'. The fourth item was answered in one of three ways, with respondents indicating that ENDS are safer than other tobacco products, that there is no difference in safety, or that ENDS are more dangerous than other tobacco products.

\section{Advertising}

Participants who had heard of ENDS were also asked whether they had been exposed to advertising for ENDS and/or had been exposed to media messages concerning the health effects of ENDS use.

\section{Self-esteem}

Participants were asked about their perceived selfesteem. The item 'I have high self-esteem' was answered using a scale 1-7; with 1 being 'Not very true of me' and 7 being 'Very true of me.'

\section{Statistical analysis}

Population characteristics, categorized by diachotomous ENDS ever use status, were compared using chi-squared tests. Simple and multiple logistic regressions were used to examine the relationship between sample population characteristics and perceptions and likelihood of ENDS ever use. A sampling weight variable was used during analytical procedures. Initial and final regression models are reported. The initial model included all of the variables previously discussed. The final model included only those variables that had a significant association at alpha $<0.05$ with the outcome variable of ENDS ever use determined using backward elimination. Backward elimination was used due to the reasonably large sample size. Results are reported significant at alpha $<0.05$. Statistical analyses were carried out using SAS statistical software (version 9.4 with SAS/ STAT 14.1, SAS Institute Inc., Cary, NG).

\section{RESULTS}

Among the youth sample population, $22.0 \%$ were aged 13 or 14 years, $34.5 \%$ were 15 or 16 years, and $43.5 \%$ were 17 or 18 years (Table 1 ). The sample was roughly equal parts male $(45.1 \%)$ and female (54.9\%), 
Table 1. Sociodemographic descriptive statistics in unweighted and weighted samples by ENDS ever use status, USA 2017

\begin{tabular}{|c|c|c|c|c|c|c|c|c|}
\hline \multirow[t]{3}{*}{ Variables } & \multicolumn{4}{|c|}{ Unweighted } & \multicolumn{4}{|c|}{ Weighted } \\
\hline & \multirow{2}{*}{$\begin{array}{c}\text { Total } \\
\text { N } 265 f^{b} \\
\text { n (\%) }\end{array}$} & \multicolumn{2}{|c|}{ EVIDS ever use } & \multirow[t]{2}{*}{$p^{d}$} & \multirow{2}{*}{$\begin{array}{c}\text { Total } \\
\mathrm{N}=250 \mathrm{I}^{\mathrm{b}} \\
\text { n }\left({ }^{\circ}{ }_{0}\right)\end{array}$} & \multicolumn{2}{|c|}{ EVDS ever use } & \multirow[t]{2}{*}{$p^{d}$} \\
\hline & & $\begin{array}{c}\text { Yes } \\
n=1617^{c} \\
n(\%)\end{array}$ & $\begin{array}{c}\text { Yo } \\
\text { n }=1037^{c} \\
\text { n }(\%)\end{array}$ & & & $\begin{array}{c}\text { Yes } \\
\text { n- } 1316^{c} \\
\text { n }(\%)\end{array}$ & $\begin{array}{c}\text { No } \\
\text { n } 1155^{c} \\
\text { n }(\%)\end{array}$ & \\
\hline Age (years) & & & & $<0.0001$ & & & & $<0.0001$ \\
\hline $13-14$ & 366 (13.8) & $148(9.2)$ & $218(21.0)$ & & $550(22.0)$ & $167(12.4)$ & $383(33.1)$ & \\
\hline $15-16$ & $670(25.2)$ & $397(24.5)$ & $273(26.3)$ & & $863(34.5)$ & $466(34.6)$ & $397(34.4)$ & \\
\hline $17-18$ & $1618(61.0)$ & $1072(66.3)$ & $546(52.7)$ & & $1088(43.5)$ & $713(53.0)$ & $375(32.5)$ & \\
\hline Gender & & & & $<0.0001$ & & & & $<0.0001$ \\
\hline Male & $1143(43.1)$ & 781 (48.3) & $362(34.9)$ & & $1128(45.1)$ & 779 (57.9) & $350(30.2)$ & \\
\hline Female & $1511(56.9)$ & $836(51.7)$ & $675(65.1)$ & & $1373(54.9)$ & $567(42.1)$ & $806(69.8)$ & \\
\hline Race/Ethnicity & & & & $<0.0001$ & & & & $<0.0001$ \\
\hline Non-Hispanic White & $1527(57.5)$ & $1025(63.4)$ & $502(48.4)$ & & $1525(61.0)$ & $889(66.0)$ & $636(55.1)$ & \\
\hline Non-Hispanic Black & $383(14.4)$ & $173(10.7)$ & $210(20.3)$ & & 333 (13.3) & $125(9.3)$ & $208(18.0)$ & \\
\hline Hispanic & $394(14.9)$ & $238(14.7)$ & $156(15.0)$ & & 473 (18.9) & $261(19.4)$ & $212(18.3)$ & \\
\hline Non-Hispanic Other & $350(13.2)$ & $181(11.2)$ & $169(16.3)$ & & $170(6.8)$ & $71(5.3)$ & $99(8.6)$ & \\
\hline Sexual orientation & & & & 0.0231 & & & & 0.0729 \\
\hline Straight & $2122(79.9)$ & $1270(78.5)$ & $852(82.2)$ & & $1980(79.2)$ & $1048(77.9)$ & $933(80.8)$ & \\
\hline LGBTO & $532(20.1)$ & $347(21.5)$ & $185(17.8)$ & & $521(20.8)$ & $298(22.1)$ & $222(19.2)$ & \\
\hline Household size (people) & & & & 0.0044 & & & & $<0.0001$ \\
\hline 1 & $92(3.5)$ & $65(4.0)$ & $27(2.6)$ & & $70(2.8)$ & $52(3.9)$ & $18(1.6)$ & \\
\hline 2 & $298(11.2)$ & $198(12.2)$ & $100(9.6)$ & & $229(9.2)$ & $135(10.0)$ & $94(8.1)$ & \\
\hline 3 & $575(21.7)$ & $377(23.3)$ & $198(19.1)$ & & $518(20.7)$ & $306(22.7)$ & $212(18.4)$ & \\
\hline 4 & $768(28.9)$ & $454(28.1)$ & $314(30.3)$ & & $739(29.5)$ & $380(28.2)$ & $359(31.1)$ & \\
\hline 5 & $485(18.3)$ & $287(17.8)$ & $198(19.1)$ & & $492(19.7)$ & $270(20.1)$ & $222(19.2)$ & \\
\hline$\geq 6$ & $436(16.4)$ & $236(14.6)$ & $200(19.3)$ & & $453(18.1)$ & $203(15.1)$ & $250(21.6)$ & \\
\hline Living status & & & & $<0.0001$ & & & & $<0.0001$ \\
\hline Both parents & $1468(55.3)$ & $812(50.2)$ & $656(63.3)$ & & $1533(61.3)$ & $751(55.8)$ & $782(67.7)$ & \\
\hline One parent & 793 (29.9) & $493(30.5)$ & $300(28.9)$ & & 733 (29.3) & $410(30.5)$ & $323(28.0)$ & \\
\hline Other guardian & $98(3.7)$ & $70(4.3)$ & $28(2.7)$ & & $85(3.4)$ & $58(4.3)$ & $27(2.3)$ & \\
\hline None of the above & $295(11.1)$ & $242(15.0)$ & $53(5.1)$ & & $150(6.0)$ & $127(9.4)$ & $23(2.0)$ & \\
\hline Lunch assistance & & & & 0.0021 & & & & 0.5570 \\
\hline Yes & $852(32.1)$ & $483(29.9)$ & $369(35.6)$ & & 894 (35.7) & $474(35.2)$ & $420(36.4)$ & \\
\hline No & $1802(67.9)$ & $1134(70.1)$ & $668(64.4)$ & & $1607(64.3)$ & $872(64.8)$ & $735(63.6)$ & \\
\hline Parental education level & & & & $<0.0001$ & & & & $<0.0001$ \\
\hline Less than high school graduate & $296(11.2)$ & $179(11.1)$ & $117(11.3)$ & & $288(11.5)$ & $157(11.7)$ & $131(11.3)$ & \\
\hline High school graduate or GED & $627(23.6)$ & $411(25.4)$ & $216(20.8)$ & & $507(20.3)$ & $304(22.6)$ & $203(17.6)$ & \\
\hline Some college & $363(13.7)$ & $254(15.7)$ & 109 (10.5) & & $365(14.6)$ & $232(17.2)$ & $133(11.5)$ & \\
\hline Associate's or technical degree & $337(12.7)$ & $226(14.0)$ & $111(10.7)$ & & $312(12.5)$ & $185(13.7)$ & $127(11.0)$ & \\
\hline Bachelor's degree & $603(22.7)$ & $338(20.9)$ & $265(25.6)$ & & $576(23.0)$ & $270(20.1)$ & $306(26.5)$ & \\
\hline Graduate or professional degree & $428(16.1)$ & $209(12.9)$ & $219(21.1)$ & & $453(18.1)$ & $198(14.7)$ & $255(22.1)$ & \\
\hline Participant education level & & & & $<0.0001$ & & & & $<0.0001$ \\
\hline Middle school or less & $314(11.8)$ & $126(7.8)$ & $188(18.1)$ & & 495 (19.8) & $157(11.7)$ & $338(29.3)$ & \\
\hline High school & $1258(47.4)$ & $723(44.7)$ & $535(51.6)$ & & $1670(66.8)$ & $940(69.8)$ & $730(63.2)$ & \\
\hline High school graduate or greater & $1082(40.8)$ & $768(47.5)$ & $314(30.3)$ & & $336(13.4)$ & $249(18.5)$ & $87(7.5)$ & \\
\hline
\end{tabular}


Table 1. Continued

\begin{tabular}{|c|c|c|c|c|c|c|c|c|}
\hline \multirow[t]{3}{*}{ Variables } & \multicolumn{4}{|c|}{ Unweighted } & \multicolumn{4}{|c|}{ Weighted } \\
\hline & \multirow{2}{*}{$\begin{array}{c}\text { Total } \\
\text { N } 26.5 t^{b} \\
\text { n }(\%)\end{array}$} & \multicolumn{2}{|c|}{ ENDS ever use } & \multirow[t]{2}{*}{$p^{d}$} & \multirow{2}{*}{$\begin{array}{c}\text { Total } \\
\text { V } 2501^{b}\end{array}$} & \multicolumn{2}{|c|}{ ENDS ever use } & \multirow[t]{2}{*}{$p^{d}$} \\
\hline & & $\begin{array}{c}\text { Yes } \\
\text { n }=1617^{c} \\
\text { n }(\%)\end{array}$ & $\begin{array}{c}\text { No } \\
\mathrm{n}=1037^{c} \\
\mathrm{n}\left({ }^{\circ}\right)\end{array}$ & & & $\begin{array}{c}\text { Yes } \\
\mathrm{n}=1316^{c} \\
\text { n }(\%)\end{array}$ & $\begin{array}{c}\text { Vo } \\
\mathrm{n}=1155^{\mathrm{c}} \\
\mathrm{n}(\%)\end{array}$ & \\
\hline Residence location & & & & 0.0971 & & & & 0.0056 \\
\hline Urban & $951(35.8)$ & $595(36.8)$ & $356(34.3)$ & & $836(33.4)$ & $479(35.6)$ & $357(30.9)$ & \\
\hline Suburban & $1119(42.2)$ & $655(40.5)$ & $464(44.8)$ & & $1080(43.2)$ & $542(40.3)$ & $538(46.6)$ & \\
\hline Rural & $584(22.0)$ & $367(22.7)$ & $217(20.9)$ & & $585(23.4)$ & $325(24.1)$ & $260(22.5)$ & \\
\hline Conventional tobacco ever use & & & & $<0.0001$ & & & & $<0.0001$ \\
\hline Yes & $1356(51.1)$ & $1227(75.9)$ & $129(12.4)$ & & $1093(43.7)$ & $977(72.6)$ & $116(10.0)$ & \\
\hline No & $1298(48.9)$ & $390(24.1)$ & $908(87.6)$ & & $1408(56.3)$ & $369(27.4)$ & 1039 (90.0) & \\
\hline
\end{tabular}

a ENDS: electronic nicotine delivery systems. b Percentages (\%) as per cent of total column N, for each variable category. c Percentages (\%) as per cent of total column $\mathrm{n}$, for each variable category. $d$ Chi-squared $p$-value for comparison between ENDS use status categories.

and $61.0 \%$ Non-Hispanic White, $13.3 \%$ Non-Hispanic Black, $18.9 \%$ Hispanic, and $6.8 \%$ Non-Hispanic Other.

Regarding tobacco use, 1346 (53.8\%) reported having ever used ENDS while 1093 (43.7\%) reported having ever used a form of conventional tobacco. Of those who had ever used a form of conventional tobacco, conventional cigarettes were the most common (75.8\%) product, followed by cigarillos (47.8\%) and hookah (41.4\%) (Table 2). Also, 977 (39.1\%) reported having ever used both ENDS and a form of conventional tobacco. Of

Table 2: Nicotine containing product ever use in weighted sample, youth aged 13-18 years, USA 2017 $(\mathrm{N}=2501)$

$\begin{array}{lcc}\text { Type of product } & \text { Yes } & \text { Vo } \\ & \left.\text { nNDS }{ }^{\mathrm{a}} \mathrm{o}\right) & \text { n }(\% \text { o } \\ \text { Cigarettes } & 1346(53.8)^{\mathrm{b}} & 1155(46.2)^{\mathrm{b}} \\ \text { Cigarillos } & 828(33.1) & 1673(66.9) \\ \text { Hookah } & 522(20.9) & 1979(79.1) \\ \text { Filtered cigars } & 453(18.1) & 2048(81.9) \\ \text { Traditional cigars } & 402(16.1) & 2099(83.9) \\ \text { Smokeless tobacco } & 363(14.5) & 2138(85.5) \\ \text { Bidis and kreteks } & 307(12.3) & 2194(87.7) \\ \text { Dissolveable tobacco } & 154(6.1) & 2347(93.9) \\ \text { Any nicotine-containing product } & 113(4.5) & 2388(95.5) \\ \text { Any conventional nicotine- } & 1093(43.7) & 1408(56.3) \\ \text { containing product } & & \\ \text { ENDS poly use }{ }^{c} & 977(39.1) & 1524(60.9)\end{array}$

a ENDS: electronic nicotine delivery systems. b Percentages (\%) as per cent of total weighted sample N. c Defined as having ever used ENDS and at least one type of conventional nicotine-containing product. those who had tried any of the nicotine-containing products discussed, ENDS were the most commonly tried first $(44.4 \%)$, followed by conventional cigarettes $(35.5 \%)$ (Table 3$)$.

Results of the initial and final binary logistic regression models are presented in Tables 4 and 5 . In the final model, for every one year increase in age, participants were 1.3 times more likely to have ever used an ENDS product (adjusted odds ratio, $\mathrm{AOR}=1.30 ; 95 \%$ CI: 1.20-1.40). Males were far more likely, 2.25 times, to have ever used ENDS than females $(\mathrm{AOR}=2.25 ; 95 \% \mathrm{CI}: 1.77-2.85)$. Participants who identified as Non-Hispanic Black were 0.57 times less likely to use ENDS than NonHispanic White participants (AOR $=0.57$; 95\% CI: 0.40-0.82). Participants who identified as Non-

Table 3. First nicotine containing product used, youth aged 13-18 years, USA $2017(\mathrm{~N}=1461)$

\begin{tabular}{lc} 
Type of product & n ( $\left.{ }^{\circ}\right)^{\text {b }}$ \\
\hline ENDS & $649(44.4)$ \\
Cigarettes & $518(35.5)$ \\
Hookah & $94(6.4)$ \\
Smokeless tobacco & $65(4.5)$ \\
Cigarillos & $60(4.1)$ \\
Traditional cigars & $31(2.1)$ \\
Filtered cigars & $16(1.1)$ \\
Dissolveable tobacco & $12(0.8)$ \\
Other & $9(0.6)$ \\
Bidis and kreteks & $7(0.5)$
\end{tabular}

a ENDS: electronic nicotine delivery systems. b Percentages (\%) as per cent of nicotine-containing product ever users in weighted sample. 
Table 4. Binary logistic regression examining association between ENDS ${ }^{\mathrm{a}}$ ever use and unweighted and weighted sample characteristics - initial model, youth aged 13-18 years, USA 2017

\begin{tabular}{|c|c|c|c|c|c|c|}
\hline \multirow[t]{2}{*}{ Variables } & \multicolumn{3}{|c|}{ Unweighted (N-2651) } & \multicolumn{3}{|c|}{ Weighted ( $=2501)$} \\
\hline & AOR & $95 \% \mathrm{CI}$ & p & AOR & $95 \% \mathrm{CI}$ & p \\
\hline Age & 1.20 & $1.07-1.35$ & 0.0018 & 1.28 & $1.16-1.43$ & $<0.0001$ \\
\hline Conventional tobacco ever use (Ref. $=$ no) & 19.68 & $15.29-25.34$ & $<0.0001$ & 19.37 & $14.80-25.35$ & $<0.0001$ \\
\hline \multicolumn{7}{|l|}{$\begin{array}{l}\text { ENDS safety comparison (Ref. = no difference in } \\
\text { safety) }\end{array}$} \\
\hline $\begin{array}{l}\text { ENDS are more dangerous than conventional } \\
\text { tobacco }\end{array}$ & 1.60 & $0.91-2.81$ & 0.1005 & 0.94 & $0.54-1.65$ & 0.8379 \\
\hline ENDS are safer than conventional tobacco & 2.94 & $2.28-3.78$ & $<0.0001$ & 2.41 & $1.85-3.14$ & $<0.0001$ \\
\hline Exposure to ENDS advertisements (Ref. = no) & 0.88 & $0.70-1.11$ & 0.2845 & 0.94 & $0.73-1.20$ & 0.6171 \\
\hline Exposure to ENDS health effects media (Ref. $=$ no) & 0.86 & $0.67-1.10$ & 0.2324 & 0.86 & $0.66-1.11$ & 0.2548 \\
\hline Gender (Ref. = female) & 2.32 & $1.80-3.01$ & $<0.0001$ & 2.40 & $1.87-3.09$ & $<0.0001$ \\
\hline Household size & 1.04 & $0.97-1.12$ & 0.3118 & 1.06 & $0.98-1.14$ & 0.1349 \\
\hline Knowledge of ENDS health risks (0-10 scale) & 1.06 & $1.02-1.10$ & 0.0026 & 1.12 & $1.07-1.16$ & $<0.0001$ \\
\hline Knowledge of tobacco health risks (0-10 scale) & 0.98 & $0.93-1.03$ & 0.3448 & 0.93 & $0.89-0.98$ & 0.0057 \\
\hline \multicolumn{7}{|l|}{ Living status (Ref. = both parents) } \\
\hline One parent & 1.26 & $0.96-1.65$ & 0.0919 & 1.23 & $0.93-1.63$ & 0.1511 \\
\hline Other guardian & 1.26 & $0.66-2.38$ & 0.4818 & 1.64 & $0.80-3.36$ & 0.1742 \\
\hline None of the above & 1.63 & $1.03-2.58$ & 0.0376 & 1.66 & $0.86-3.20$ & 0.1310 \\
\hline Lunch assistance $($ Ref. $=$ no) & 0.69 & $0.53-0.90$ & 0.0055 & 0.69 & $0.53-0.92$ & 0.0097 \\
\hline \multicolumn{7}{|l|}{$\begin{array}{l}\text { Parent education level (Ref. = high school } \\
\text { graduate or GED) }\end{array}$} \\
\hline Less than high school graduate & 0.73 & $0.48-1.10$ & 0.1328 & 0.52 & $0.33-0.82$ & 0.0046 \\
\hline Some college & 1.33 & $0.90-1.97$ & 0.1551 & 1.22 & $0.81-1.84$ & 0.3302 \\
\hline Associate's or technical degree & 1.27 & $0.86-1.89$ & 0.2335 & 0.99 & $0.65-1.50$ & 0.9625 \\
\hline Bachelor's degree & 0.70 & $0.50-0.98$ & 0.0386 & 0.49 & $0.34-0.71$ & 0.0001 \\
\hline Graduate or professional degree & 0.51 & $0.35-0.74$ & 0.0004 & 0.49 & $0.33-0.82$ & 0.0003 \\
\hline \multicolumn{7}{|l|}{$\begin{array}{l}\text { Participant education level (Ref. = middle school } \\
\text { or less) }\end{array}$} \\
\hline High school & 1.11 & $0.72-1.72$ & 0.6462 & 1.03 & $0.69-1.53$ & 0.8817 \\
\hline High school graduate or greater & 0.95 & $0.54-1.69$ & 0.8647 & 0.90 & $0.49-1.65$ & 0.7355 \\
\hline $\begin{array}{l}\text { Perception of how dangerous or safe ENDS are } \\
\text { (0-10 scale) }\end{array}$ & 1.24 & $1.18-1.29$ & $<0.0001$ & 1.26 & $1.21-1.32$ & $<0.0001$ \\
\hline \multicolumn{7}{|l|}{ Race/Ethnicity (Ref. = Non-Hispanic White) } \\
\hline Non-Hispanic Black & 0.53 & $0.38-0.75$ & 0.0003 & 0.56 & $0.39-0.81$ & 0.0024 \\
\hline Hispanic & 0.74 & $0.53-1.03$ & 0.0764 & 0.78 & $0.56-1.07$ & 0.1240 \\
\hline Non-Hispanic Other & 0.80 & $0.58-1.12$ & 0.1953 & 0.57 & $0.36-0.92$ & 0.0215 \\
\hline Self-reported self-esteem (1-7 scale) & 1.03 & $0.97-1.10$ & 0.3602 & 0.99 & $0.93-1.06$ & 0.8663 \\
\hline Sexual orientation (Ref. = straight) & 1.32 & $0.99-1.77$ & 0.0591 & 1.26 & $0.93-1.70$ & 0.1320 \\
\hline \multicolumn{7}{|l|}{ Residence location (Ref. = suburban) } \\
\hline Urban & 1.02 & $0.79-1.32$ & 0.8852 & 1.00 & $0.76-1.32$ & 0.9925 \\
\hline Rural & 0.86 & $0.63-1.17$ & 0.3293 & 0.87 & $0.64-1.20$ & 0.4020 \\
\hline
\end{tabular}

a ENDS: electronic nicotine delivery systems. AOR: adjusted odds ratio. Cl: confidence interval. 
Table 5. Binary logistic regression examining association between ENDS a ever use and unweighted and weighted sample characteristics - final model, youth aged 13-18 years, USA 2017

\begin{tabular}{|c|c|c|c|c|c|c|}
\hline \multirow[t]{2}{*}{ Variables } & \multicolumn{3}{|c|}{ Unweighted ( $\mathrm{V}=2651)$} & \multicolumn{3}{|c|}{ Weighted ( $\mathrm{N}=2501)$} \\
\hline & AOR & $95 \% \mathrm{CI}$ & p & AOR & $95 \% \mathrm{CI}$ & $p$ \\
\hline Age & 1.21 & $1.12-1.30$ & $<0.0001$ & 1.30 & $1.20-1.40$ & $<0.0001$ \\
\hline Conventional tobacco ever use (Ref. $=$ no) & 20.23 & 15.77-25.96 & $<0.0001$ & 19.96 & 15.30-26.05 & $<0.0001$ \\
\hline \multicolumn{7}{|l|}{$\begin{array}{l}\text { ENDS safety comparison (Ref. = no difference in } \\
\text { safety) }\end{array}$} \\
\hline $\begin{array}{l}\text { ENDS are more dangerous than conventional } \\
\text { tobacco }\end{array}$ & 1.50 & $0.86-2.60$ & 0.1540 & 0.90 & $0.52-1.56$ & 0.7163 \\
\hline ENDS are safer than conventional tobacco & 2.82 & $2.20-3.62$ & $<0.0001$ & 2.37 & $1.82-3.08$ & $<0.0001$ \\
\hline Gender (Ref. = female) & 2.24 & $1.75-2.87$ & $<0.0001$ & 2.25 & $1.77-2.85$ & $<0.0001$ \\
\hline Knowledge of ENDS health risks (0-10 scale) & 1.05 & $1.01-1.09$ & 0.0068 & 1.11 & $1.06-1.15$ & $<0.0001$ \\
\hline Knowledge of tobacco health risks (0-10 scale) & 0.98 & $0.94-1.03$ & 0.4084 & 0.93 & $0.89-0.98$ & 0.0061 \\
\hline Lunch assistance (Ref. $=$ no) & 0.73 & $0.57-0.94$ & 0.0157 & 0.75 & $0.58-0.97$ & 0.0341 \\
\hline \multicolumn{7}{|l|}{$\begin{array}{l}\text { Parent education level (Ref. = high school } \\
\text { graduate or GED) }\end{array}$} \\
\hline Less than high school graduate & 0.74 & $0.49-1.12$ & 0.1417 & 0.54 & $0.35-0.84$ & 0.0064 \\
\hline Some college & 1.31 & $0.89-1.94$ & 0.1687 & 1.20 & $0.80-1.80$ & 0.3731 \\
\hline Associate's or technical degree & 1.26 & $0.85-1.85$ & 0.2526 & 0.96 & $0.64-1.45$ & 0.8607 \\
\hline Bachelor's degree & 0.69 & $0.50-0.96$ & 0.0254 & 0.48 & $0.34-0.69$ & $<0.0001$ \\
\hline Graduate or professional degree & 0.50 & $0.35-0.72$ & 0.0002 & 0.49 & $0.34-0.70$ & 0.0001 \\
\hline $\begin{array}{l}\text { Perception of how dangerous or safe ENDS are } \\
\text { (0-10 scale) }\end{array}$ & 1.24 & $1.19-1.30$ & $<0.0001$ & 1.26 & $1.21-1.32$ & $<0.0001$ \\
\hline \multicolumn{7}{|l|}{ Race/Ethnicity (Ref. = Non-Hispanic White) } \\
\hline Non-Hispanic Black & 0.57 & $0.41-0.79$ & 0.0007 & 0.57 & $0.40-0.82$ & 0.0022 \\
\hline Hispanic & 0.77 & $0.56-1.07$ & 0.1184 & 0.79 & $0.58-1.07$ & 0.1334 \\
\hline Non-Hispanic Other & 0.82 & $0.59-1.14$ & 0.2432 & 0.58 & $0.37-0.93$ & 0.0248 \\
\hline
\end{tabular}

a ENDS: electronic nicotine delivery systems. AOR: adjusted odds ratio. $\mathrm{Cl}$ : confidence interval.

Hispanic Other were also less likely (i.e. 0.58 times) to use ENDS than Non-Hispanic White participants (AOR=0.58; 95\% CI: 0.37-0.93). Those participants whose parents were either in the lowest education category or the highest two education categories were less likely to have ever used ENDS compared to those in the reference group of high school graduate/GED recipients.

Not surprisingly, those who had ever used some form of conventional tobacco were far more likely to have ever used an ENDS product as well (AOR=19.96; 95\% CI: 15.30-26.05). Participants' comparative knowledge of conventional tobacco and ENDS use safety, as well as health effects of these products, was found to be significantly associated with the likelihood of ever using ENDS. For every one unit increase on the knowledge of tobacco health risks ordinal scale, participants were 0.93 times less likely to have ever used an ENDS product (AOR=0.93; 95\% CI: 0.89-0.98). Interestingly, for every one unit increase on the knowledge of ENDS health risks ordinal scale, participants were 1.11 times more likely to have ever used ENDS (AOR $=1.11 ; 95 \%$ CI: 1.06-1.15). Participants who believed ENDS to be safe were 1.26 times more likely to have ever used ENDS for every one unit increase on the perceived safety of ENDS ordinal scale $(\mathrm{AOR}=1.26 ; 95 \% \mathrm{CI}: 1.21-1.32)$. Similarly, those who believed that ENDS were safer than conventional tobacco were much more likely to have ever used ENDS (AOR=2.37; 95\% CI: 1.82-3.08).

\section{DISCUSSION}

These findings suggest that certain sections of 
the US youth population are at greater risk for initiation of ENDS use than others. As research on the health outcomes of ENDS use continues, it is vital to emphasize the importance of education and prevention campaigns and to know which groups may benefit most from these efforts in order to maximize impact.

Study findings indicate that age is a significant factor in determing the likelihood of ENDS ever use, with the odds of ever use substantially increased for every one year increase in age. Thus, by the time US youth reach the age of 18 years, their odds of having ever used an ENDS product are quite high. This finding also suggests that education and prevention campaigns might be most effective when introduced earlier rather than later in adolesence. Exposure to anti-tobacco media campaigns in younger students has been shown to result in reduced cigarette and smokeless tobacco use, which suggests a similar outcome may be achievable with ENDS prevention ${ }^{26}$. ENDS use is increasingly pervasive within the US youth population, and exposure to these products is undoubtedly high during middle and high school, likely compelling many non-users to initiate use as personal and social pressures mount.

In addition to age, other sociodemographic factors, including gender, parental education level, and race/ethnicity, are also significantly associated with likelihood of ENDS initiation. Males are much more likely to be ever users than females. Participants whose parents are either not high school graduates or who hold a Bachelor's or higher academic degree are less likely to be ever users. Participants who identify as Non-Hispanic Black or Non-Hispanic Other are less likely to be ever users as well. Modeling also revealed that those who report utilizing lunch assistance programs, a potential proxy for familial socioeconomic status (SES), may be less likely to initiate ENDS use. These sociodemographic factors are important considerations as tailored education and prevention messages are developed.

Our findings on associations between SES and ENDS use warrant investigaton in future research. In this study, participants using lunch assistance programs and those whose parents did not complete high school, both potential proxies that might indicate lower SES, as well as those whose parents had a Bachelor's or higher degree were less likley to use ENDS. Although past examinations of SES and tobacco use are not conclusive, some evidence suggests associations between higher SES and elevated ENDS use, as well as between lower SES, higher combustible cigarette use, and lower ENDS use ${ }^{27,28}$. It is possible that the likelihood of using ENDS products is shaped by different factors for these groups of youth. For example, perhaps youth who may be lower in SES make choices based on disposible income. It may be easier to procure traditional tobacco products because they may cost less than ENDS (e.g. buying a 'loosie') ${ }^{29}$. Alternatively, tobacco use patterns of family members or friends may shape the product choices of youth or the products that they have most easily available to them. That is, if the family members of lower SES youth are more likely to smoke cigarettes, then they may be more likely to try combustible tobacco and less likely to use ENDS. Our finding that youth with college-educated parents are less likely to use ENDS is surprising, as it differs with some past research findings showing connections between higher income and ENDS use. One possibility is that parent education level may not be a good indicator of SES (e.g. student debt rising, wages stagnating). Another possibility might be that awareness has increased among higher income parents, lower income parents, or both groups, and that they are talking with their children more about these products and/or monitoring their use.

Ever use of conventional tobacco products is significantly associated with a substantial increase in odds of being an ENDS ever user. Those who are least familiar with the health risks associated with conventional tobacco use are also most likely to be ever users of ENDS. As our study results underscore, harm perceptions such as these are highly important. Participants reporting that ENDS are safer than conventional tobacco products have significantly greater odds of being ENDS ever users. Similarly, those reporting that ENDS are 'very safe' as opposed to 'very dangerous' are significantly more likely to have initiated ENDS use at some point in their lives. Interestingly, those indicating that they are most familiar with the health risks associated with ENDS use are also most likely to be ever users of these products. Actual ENDS health-risk knowledge held by participants was not tested, so it is possible that 
those who felt they knew more about the health risks of ENDS also felt that they were better equiped to evaluate the safety of these products, and ultimately concluded that they were safe in many cases. The potential for misinformation leading to a perceived sense of elevated ENDS health risk knowledge in ENDS ever users is a strong supporting factor for education and prevention campaigns that highlight the current body of literature surrounding ENDS health-risk data.

In our sample, ENDS were the most common first-used nicotine-containing product, followed by conventional cigarettes. As perceptions of knowledge and safety of nicotine-containing products were significantly associated with likelihood of ENDS use, it seems possible that topic-related information deficiencies may be partially to blame for escalating patterns of use. When this information is coupled with the noted association between increasing age and ENDS ever use, introducing education and prevention campaigns early in middle school or prior to middle school seems warranted.

Overall, the results support the existing literature suggesting that those youth at greatest risk for ENDS use are Non-Hispanic White males in their late teens ${ }^{22-25}$, and that ENDS are surpassing conventional tobacco products as the predominant nicotine-containing product used by US youth ${ }^{5,6,9,30}$. Paralleling findings in other studies, Non-Hispanic Black youth in our sample were generally less likely to use $\operatorname{ENDS}^{31,32}$. Also consistent with the existing literature was the finding that perceiving ENDS to be safe or safer than conventional tobacco products increased the likelihood of ENDS ever use ${ }^{33}$. Though examining ever use behaviors does not allow temporality assessment, the association between conventional tobacco ever use and ENDS ever use in our study is consistent with the literature suggesting ENDS use may increase the likelihood of uptake of a conventional tobacco habit in former non-users or users of both types of products ${ }^{34-36}$.

\section{Limitations}

When evaluating the findings, it is important to note the limitations associated with the study. First, its cross-sectional design negated the possibility of assessing the effects of temporality on the observed relationships. Second, the sample was recruited non- randomly, as certain subgroups were oversampled to ensure that adequate comparisons could be made, and the questionnaire was adminstered online; thus, qualified individuals lacking sufficient Internet access may have been excluded. Third, recall bias may have influenced self-reported responses, which, for example, may have led to under-reporting of nicotine product usage (e.g. if such use was not recent). Fourth, sample size was reduced in some cases due to missing or incomplete data. Also, after removal of missing or incomplete data, only participants who identified as male or female remained in the analysis; thus, persons who identify with other gender identities may not be represented by these findings.

\section{CONCLUSIONS}

The study findings suggest that certain US youth subpopulations have an elevated likelihood of being ENDS ever users. The subpopulation at greatest risk for ever use of ENDS appears to be Non-Hispanic White males in their mid to late teens. Furthermore, perceptions of the health effects and safety of nicotinecontaining products are significantly related to odds of ENDS ever use. With increasing evidence suggesting potential harms associated with ENDS use and the prevalence of ENDS use steadily rising in the US, prevention efforts may consider precisely targeting groups most vulnerable to use, as well as starting educational initiatives at or prior to middle school. Prevention campaigns coupled with increasingly stringent regulations on the marketing and sale of ENDS being implemented by the US Food and Drug Administration (FDA) will likely prove beneficial. Future research efforts are needed to further examine why certain groups are more susceptible than others.

\section{REFERENCES}

1. Farrelly MC, Healton CG, Davis KC, Messeri P, Hersey JC, Haviland ML. Getting to the truth: Evaluating national tobacco countermarketing campaigns. Am J Public Health. 2002;92(6):901-907. doi:10.2105/ajph.92.6.901

2. Murphy-Hoefer R, Davis KC, Beistle D, et al. Impact of the Tips from Former Smokers campaign on populationlevel smoking cessation, 2012-2015. Prev Chronic Dis. 2018;15. doi:10.5888/pcd15.180051

3. Farrelly MC, Duke JC, Nonnemaker J, et al. Association between The Real Cost media campaign and smoking initiation among youths-United States, 2014-2016. MMWR Morb Mortal Wkly Rep. 2017;66(02):47-50. doi:10.15585/mmwr.mm6602a2 
4. Centers for Disease Control and Prevention. Fast Facts: Smoking \& Tobacco Use, 2019. https://www.cdc.gov/ tobacco/data_statistics/fact_sheets/fast_facts/index.htm. Published February 6, 2019. Accessed August 9, 2019.

5. Centers for Disease Control and Prevention. Smoking is down, but almost 38 million American adults still smoke. https://www.cdc.gov/media/releases/2018/p0118smoking-rates-declining.html. Published January 18, 2018. Accessed July 31, 2019.

6. Centers for Disease Control and Prevention. Trends in Current Cigarette Smoking Among High School Students and Adults, United States, 1965-2014. https://www.cdc. gov/tobacco/data_statistics/tables/trends/cig_smoking/ index.htm. Accessed July 31, 2019.

7. Gentzke A, Creamer M, Cullen K, et al. Vital Signs: Tobacco product use among middle and high school studentsUnited States, 2011-2018. MMWR Morb Mortal Wkly Rep. 2019(68):157-164. doi:10.15585/mmwr.mm6806e1

8. Cullen K, Ambrose B, Gentzke A, Apelberg BJ, Jamal A, King B. Notes from the field: Use of electronic cigarettes and any tobacco product among middle and high school students-United States, 2011-2018. MMWR Morb Mortal Wkly Rep. 2018;67(45):1276-1277. doi:10.15585/mmwr.mm6745a5

9. Walley SC, Jenssen BP, Section on Tobacco Control. Electronic nicotine delivery systems. Pediatrics. 2015;136(5):1018-1026. doi:10.1542/peds.2015-3222

10. Centers for Disease Control and Prevention. Notes from the field: Electronic cigarette use among middle and high school students-United States, 2011-2012. MMWR Morb Mortal Wkly Rep. 2013;62(35):729-730. https://www. cdc.gov/mmwr/preview/mmwrhtml/mm6235a6.htm. Published September, 2013. Accessed October 13, 2019.

11. Essenmacher C, Naegle M, Baird C, et al. Electronic nicotine delivery systems (ENDS): What nurses need to know. J Am Psychiatr Nurses Assoc. 2018;24(2):145-152. doi:10.1177/1078390317733802

12. Wagoner KG, Cornacchione J, Wiseman KD, Teal R, Moracco KE, Sutfin EL. E-cigarettes, hookah pens and vapes: Adolescent and young adult perceptions of electronic nicotine delivery systems. Nicotine Tob Res. 2016;18(10):2006-2012. doi:10.1093/ntr/ntw095

13. Oh AY, Kacker A. Do electronic cigarettes impart a lower potential disease burden than conventional tobacco cigarettes? Review on e-cigarette vapor versus tobacco smoke. Laryngoscope. 2014;124(12):2702-2706. doi:10.1002/lary.24750

14. Caponnetto P, Polosa R, Russo C, Leotta C, Campagna D. Successful smoking cessation with electronic cigarettes in smokers with a documented history of recurring relapses: A case series. J Med Case Rep. 2011;5(1):585. doi:10.1186/1752-1947-5-585

15. Barbeau AM, Burda J, Siegel M. Perceived efficacy of e-cigarettes versus nicotine replacement therapy among successful e-cigarette users: A qualitative approach. Addict
Sci Clin Pract. 2013;8(1):5. doi:10.1186/1940-0640-8-5

16. Olmedo P, Goessler W, Tanda S, et al. Metal concentrations in e-cigarette liquid and aerosol samples: The contribution of metallic coils. Environ Health Perspect. 2018;126(2):027010. doi:10.1289/EHP2175

17. Dutra LM, Glantz SA. Electronic cigarettes and conventional cigarette use among U.S. adolescents: A cross-sectional study. JAMA Pediatr. 2014;168(7):610617. doi:10.1001/jamapediatrics.2013.5488

18. Hajek P, Phillips-Waller A, Przulj D, et al. A randomized trial of e-cigarettes versus nicotine-replacement therapy. N Engl J Med. 2019;380(7):629-637. doi:10.1056/nejmoa1808779

19. Bold KW, Kong G, Camenga DR, et al. Trajectories of e-cigarette and conventional cigarette use among youth. Pediatrics. 2018;141(1):e20171832. doi:10.1542/peds.2017-1832

20. Siegel DA, Jatlaoui TC, Koumans EH, et al. Update: Interim guidance for health care providers evaluating and caring for patients with suspected e-cigarette, or vaping, product use associated lung injury - United States, October 2019. MMWR Morb Mortal Wkly Rep. 2019(68):919-927. doi:10.15585/mmwr.mm6841e3

21. Timberlake DS, Nikitin D, Garcia-Cano J, Cino S, Savkina M, Pechmann C. Linking the content to demographic reach of online advertising of electronic nicotine delivery systems. Tob Control. 2018;27(4):463-469. doi:10.1136/tobaccocontrol-2016-053473

22. Arrazola RA, Singh T, Corey CG, et al. Tobacco use among middle and high school students-United States, 20112014. MMWR Morb Mortal Wkly Rep. 2015;64(14):381385. https://www.cdc.gov/mmwr/preview/mmwrhtml/ mm6414a3.htm. Published April, 2015. Accessed October $13,2019$.

23. Anand V, McGinty KL, O'Brien K, Guenthner G, Hahn E, Martin CA. E-cigarette use and beliefs among urban public high school students in North Carolina. J Adolesc Health. 2015;57(1):46-51. doi:10.1016/j.jadohealth.2015.03.018

24. Bostean G, Trinidad DR, McCarthy WJ. E-cigarette use among never-smoking California students. Am J Public Health. 2015;105(12):2423-2425. doi:10.2105/AJPH.2015.302899

25. Krishnan-Sarin S, Morean ME, Camenga DR, Cavallo DA, Kong G. E-cigarette use among high school and middle school adolescents in Connecticut. Nicotine Tob Res. 2015;17(7):810-818. doi:10.1093/ntr/ntu243

26. Cavazos-Rehg PA, Krauss MJ, Sowles SJ, et al. Multiple levels of influence that impact youth tobacco use. Tob Regul Sci. 2016;2(2):106-122. doi:10.18001/trs.2.2.2

27. Harlow AF, Stokes A, Brooks DR. Socioeconomic and racial/ethnic differences in e-cigarette uptake among cigarette smokers: Longitudinal analysis of the Population Assessment of Tobacco and Health (PATH) Study. Nicotine Tob Res. 2019;21(10):1385-1393. doi:10.1093/ntr/nty141 
28. Nandi A, Glymour MM, Subramanian SV. Association among socioeconomic status, health behaviors, and allcause mortality in the United States. Epidemiology. 2014;25(2):170-177. doi:10.1097/ede.0000000000000038

29. Von Lampe K, Kurti M, Johnson J. 'I'm gonna get me a loosie' understanding single cigarette purchases by adult smokers in a disadvantaged section of New York City. Prev Med Rep. 2018;12:182-185. doi:10.1016/j.pmedr.2018.09.016

30. Wang T, Gentzke A, Sharapova S, Cullen K, Ambrose B, Jamal A. Tobacco product use among middle and high school students-United States 2011-2017. MMWR Morb Mortal Wkly Rep. 2018;67(22):629-633. doi:10.15585/mmwr.mm6722a3

31. Hess C, Antin T, Annechino R, Hunt G. Perceptions of e-cigarettes among black youth in California. Int J Environ Res and Public Health. 2017;14(1):60. doi:10.3390/ijerph14010060

32. Cooper M, Case KR, Loukas A. E-cigarette use among Texas youth: Results from the 2014 Texas youth tobacco survey. Addict Beh. 2015;50:173-177. doi:10.1016/j.addbeh.2015.06.034

33. Ambrose BK, Rostron BL, Johnson SE, et al. Perceptions of the relative harm of cigarettes and e-cigarettes among U.S. youth. Am J Prev Med. 2014;47(2):S53-S60. doi:10.1016/j.amepre.2014.04.016

34. Glantz SA, Bareham DW. E-cigarettes: Use, effects on smoking, risks, and policy implications. Annu Rev Public Health. 2018;39(1):215-235. doi:10.1146/annurev-publhealth-040617-013757

35. National Academies of Sciences, Engineering, and Medicine. Public health consequences of e-cigarettes. Washington, DC: The National Academies Press; 2018;493-540. doi:10.17226/24952

36. Soneji S, Barrington-Trimis JL, Wills TA, et al. Association between initial use of e-cigarettes and subsequent cigarette smoking among adolescents and young adults. JAMA Pediatr. 2017;171(8):788. doi:10.1001/jamapediatrics.2017.1488
ACKNOWLEDGEMENTS

We extend our thanks to Shesh Rai for assistance with data analysis.

CONFLICTS OF INTEREST

The authors have each completed and submitted an ICMJE form for disclosure of potential conflicts of interest. The authors declare that they have no competing interests, financial or otherwise, related to the current work. All authors report grants from the National Institutes of Health and the Food and Drug Administration during the conduct of the study.

\section{FUNDING}

This work was supported by the National Heart, Lung, and Blood Institute (NHLBI) of the National Institutes of Health (NIH) and the Food and Drug Administration's Center for Tobacco Products (CTP) under Award Numbers P50HL120163 and U54HL120163. The content is solely the responsibility of the authors and does not necessarily represent the official views of the NIH, the Food and Drug Administration, or the American Heart Association.

PROVENANCE AND PEER REVIEW

Not commissioned; externally peer reviewed. 